\title{
Impact of Extrahepatic Metastases on Overall Survival in Patients with Advanced Liver Dominant Hepatocellular Carcinoma: A Subanalysis of the SORAMIC Trial
}

\author{
Kerstin Schütte ${ }^{a, b}$ Regina Schinner ${ }^{c}$ Mathias P. Fabritius ${ }^{c}$ \\ Melina Möller ${ }^{c}$ Christiane Kuhl $^{d}$ Roberto lezzi ${ }^{\mathrm{e}}$ Osman Öcal ${ }^{c}$ \\ Maciej Pech ${ }^{f}$ Bora Peynircioglug Max Seidensticker ${ }^{c}$ Rohini Sharmah \\ Daniel Palmer ${ }^{i}$ Jean-Pierre Bronowickij Peter Reimerk \\ Peter Malfertheinerl Jens Ricke ${ }^{c}$

\begin{abstract}
aDepartment of Gastroenterology, Hepatology and Infectious Diseases, Otto-von-Guericke University, Magdeburg, Germany; ${ }^{b}$ Department of Internal Medicine and Gastroenterology, Niels-Stensen-Kliniken Marienhospital, Osnabrück, Germany; ' $D$ epartment of Radiology, Hospital of the Ludwig Maximilian University of Munich, Munich, Germany; ${ }^{d}$ Department of Radiology, Universitätsklinikum Aachen, Aachen, Germany; eDipartimento di Diagnostica per Immagini, Radioterapia Oncologica ed Ematologia - Istituto di Radiologia, Fondazione Policlinico Universitario A. Gemelli IRCCS, Rome, Italy; ${ }^{f}$ Department of Radiology and Nuclear Medicine, Otto-von-Guericke University, Magdeburg, Germany; ${ }^{9}$ Department

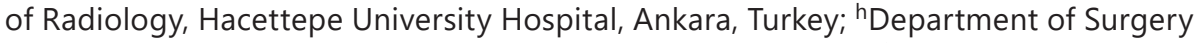
\& Cancer, Imperial College Healthcare NHS Trust, London, UK; 'Molecular and Clinical Cancer Medicine, University Hospitals \& Clatterbridge, University of Liverpool, Liverpool, UK; jDepartment of Hepatology, INSERM U1254, Hôpital de Brabois, CHU de Nancy, University of Lorraine, Nancy, France; ${ }^{k}$ Institute for Diagnostic and Interventional Radiology, Städtisches Klinikum Karlsruhe, Karlsruhe, Germany; 'Department of Internal Medicine II, Hospital of the Ludwig Maximilian University of Munich, Munich, Germany
\end{abstract}

\section{Keywords}

Hepatocellular carcinoma $\cdot$ Prognosis · Metastases · Sorafenib

\begin{abstract}
Introduction: Extrahepatic spread is reported as a prognostic factor in patients with advanced hepatocellular carcinoma (HCC) receiving systemic therapy. However, clinical studies have reported conflicting results for the clinical impact of the pattern of tumor progression during treatment and the role of new extrahepatic metastases in length of survival. Objective: To evaluate the impact of extrahepatic metastases on survival in patients with HCC treated with sorafenib or with a combination of sorafenib and selective internal radiation treatment
\end{abstract}


(SIRT). Methods: SORAMIC is a randomized, controlled trial comprising diagnostic, local ablation, and palliative cohorts. In the palliative cohort, patients not eligible for transarterial chemoembolization (TACE) were randomized 11:10 to SIRT plus sorafenib (SIRT + sorafenib) or sorafenib alone. This exploratory subanalysis evaluated the impact of extrahepatic metastases on survival. Results: In the intent-to-treat cohort, 216 patients were randomized to SIRT + sorafenib and 208 to sorafenib alone. Seventeen patients with distant organ metastases (bone, $n=11$; adrenal glands, $n=5$; peritoneum, $n=1$ ) and 262 without distant metastases at study entry were analyzed in this substudy. Patients with (Group A) and without (Group B) distant organ metastases at study entry presented with a median survival of 11.3 and 14.8 months, respectively ( $p=0.2807$ ). During follow-up of patients with no organ metastases at baseline, extrahepatic disease progression occurred in 50 patients (19.1\%). No statistically significant difference in survival was observed between patients without extrahepatic progression and those with new extrahepatic disease during treatment (14.8 vs. 14.9 months; $p=$ 0.6483 ). Development of new pulmonary metastases during treatment significantly shortened median survival ( 7.6 vs. 15.0 months, $p=0.0060$ ). Conclusions: This subanalysis of the SORAMIC trial suggests that in patients with liver-dominant advanced HCC, metastases to distant organs with the exception of pulmonary metastases do not in general exert a negative impact on patient prognosis. The choice of palliative treatment should incorporate a personalized analysis of the pattern of tumor distribution.

(C) 2020 The Author(s).

Published by S. Karger AG, Basel

\section{Introduction}

Hepatocellular carcinoma (HCC) represents the second most common cause of cancerrelated deaths. Hepatitis $\mathrm{C}$ and $\mathrm{B}$ virus infections continue to drive the burden of HCC in developing countries [1], whereas in developed countries, alcoholic liver cirrhosis and increasing epidemics of nonalcoholic steatohepatitis are the predominant factors responsible for the incidence of HCC [2-4].

In patients with HCC, factors related to the tumor itself, liver function, and clinical performance status influence survival. The majority of patients with HCC are diagnosed in advanced tumor stages. The standard of care in intermediate-stage HCC according to the Barcelona Clinic Liver Cancer (BCLC) classification is transarterial chemoembolization (TACE) [5]. Patients with large tumor or portal vein thrombosis are not suitable for TACE for technical reasons and are offered radioembolization with selective internal radiation treatment (SIRT) or systemic therapy $[5,6]$. In patients with advanced disease, treatment with the multikinase inhibitor, sorafenib, has been the standard of care since 2008; sorafenib prolongs overall survival but does not result in radiological objective response [7]. Except for select subgroups, SIRT in combination with sorafenib did not provide superior benefit compared to either type of therapy alone in our recent trial of patients with advanced HCC [8].

It remains uncertain whether tumor control impeding tumor progression or preservation of liver function is the key factor in survival prolongation. Effective treatment of the primary in the liver has the potential to provide both effects, as deterioration of liver function is frequently a consequence of intrahepatic tumor progression.

Distant metastases are depicted by imaging at the time of diagnosis in $13-36 \%$ of patients with HCC, while the prevalence of distant metastases reaches up to 68\% in autopsy studies [9-11]. Extrahepatic spread has been reported as a prognostic factor in patients with advanced disease receiving sorafenib [12]. However, the clinical impact of the pattern of tumor progression during treatment and the role of new extrahepatic metastases in the length of survival are unclear. Clinical studies have reported conflicting results $[13,14]$.

\section{Karger'}


While analyses of well-characterized and prospectively followed patients with HCC receiving sorafenib treatment suggest that new extrahepatic metastases, developed over the course of treatment, have a significant impact on survival $[13,15,16]$, other studies emphasize the important role of intrahepatic tumor control even in patients with metastases [17-19].

SORAfenib in combination with local MICrotherapy guided by gadolinium-EOB-DTPAenhanced MRI (SORAMIC) (EudraCT 2009-012576-27, NCT01126645) is a prospective study that comprises 3 substudies: (i) comparison of Gd-EOB-DTPA (gadoxetic acid; Primovist ${ }^{\circledR}$, Eovist ${ }^{\circledR}$ )-enhanced magnetic resonance imaging (MRI) versus contrast-enhanced multislice computed tomography for the stratification of patients to a local ablation (curative treatment) or palliative treatment group; (ii) comparison of radiofrequency ablation (RFA) plus sorafenib versus control (RFA plus matching placebo) on time to recurrence; and (iii) comparison of SIRT with yttrium-90 $\left({ }^{90} \mathrm{Y}\right)$ resin microspheres combined with sorafenib with control (sorafenib alone) on overall survival [8]. The aim of our current study was to evaluate the impact of extrahepatic metastases on survival in patients with HCC treated with sorafenib or with a combination of sorafenib and SIRT within this randomized multicenter study.

\section{Methods}

The randomized, controlled, multicenter phase II SORAMIC study in its palliative treatment arm aimed - among other primary objectives - to evaluate the impact of ${ }^{90} \mathrm{Y}$ microspheres (SIRT) combined with systemic therapy using sorafenib compared to sorafenib alone on survival in patients with advanced HCC. The primary results have been published recently [8].

Patients aged 18-85 years with a diagnosis of HCC were eligible for the palliative arm of the SORAMIC trial in case of liver-dominant disease BCLC B or C and liver function in Child-Pugh stage A or B up to 7 points, if they were ineligible for TACE and after providing written informed consent to participate in the study. Metastases to bone, lymph nodes, and adrenal glands at baseline did not constitute exclusion criteria, but all other sites of extrahepatic spread impeded inclusion in the trial. The primary study endpoint in this part of the study was overall survival.

Patients were randomized in 11:10 ratio to receive either systemic therapy with sorafenib or SIRSpheres therapy in combination with sorafenib. Patients randomized but in whom radioembolization could not be performed for technical reasons remained in the study and were switched to the sorafenib-only arm. In patients with HCC nodules in both liver lobes, radioembolization was initially performed on the dominantdiseased liver lobe, followed by radioembolization to the untreated contralateral lobe 4-6 weeks later. On day 3 after the last radioembolization, systemic treatment with sorafenib was initiated at a dose of $200 \mathrm{mg}$ twice daily that was escalated, if tolerated, to $400 \mathrm{mg}$ twice daily after 1 week.

Patients were followed at 2-month intervals by clinical assessment, assessment of adverse events, and laboratory workup for at least 2 years or until death; diagnostic imaging was not required in the palliative group in the context of the SORAMIC trial but was performed at the discretion of local investigators. If diagnostic imaging was performed during follow-up, results were to be reported on the case report form. Patients who needed to discontinue sorafenib remained on the study.

In the intent-to-treat (ITT) cohort of the SORAMIC trial, 216 patients were randomized to SIRT + sorafenib and 208 to sorafenib alone. Median overall survival was 12.1 months in the SIRT + sorafenib arm and 11.4 months in the sorafenib arm (hazard ratio [HR] 1.01, 95\% confidence interval [CI] 0.81-1.25; $p=$ 0.9529). Median overall survival in the per-protocol (PP) population was 14.0 months in the SIRT + sorafenib $\operatorname{arm}(n=114)$ and 11.1 months in the sorafenib arm $(n=174 ;$ HR $0.86 ; p=0.2515)$. Subgroup analyses of the PP population indicated a survival benefit for SIRT + sorafenib in patients without cirrhosis (HR 0.46, 95\% CI 0.25-0.86; $p=0.02$ ), cirrhosis of nonalcoholic etiology (HR 0.63, 95\% CI 0.45-0.89; $p=0.012$ ), or age $\leq 65$ years (HR 0.65, 95\% CI 0.43-1.0; $p=0.05$ ) [8].

The exploratory subanalysis presented here evaluated the impact of extrahepatic metastases beyond metastases to the lymph nodes on survival in these patients. Patients were included in this analysis if they received study treatment within the palliative arm of SORAMIC and were followed up with imaging. Of the 424 patients randomized after assignment to the palliative arm, 279 fulfilled these criteria within the ITT population, comprising 147 patients treated with sorafenib alone, 129 patients treated with the combination 


\begin{tabular}{|c|c|c|}
\hline \multirow[b]{2}{*}{ Liver Cancer } & \multicolumn{2}{|c|}{ Liver Cancer 2020;9:771-786 } \\
\hline & DOI: 10.1159/000510798 & $\begin{array}{l}\text { (c) } 2020 \text { The Author(s). Published by S. Karger AG, Basel } \\
\text { www.karger.com/lic }\end{array}$ \\
\hline
\end{tabular}

Schütte et al.: Impact of Metastasis on Survival in HCC

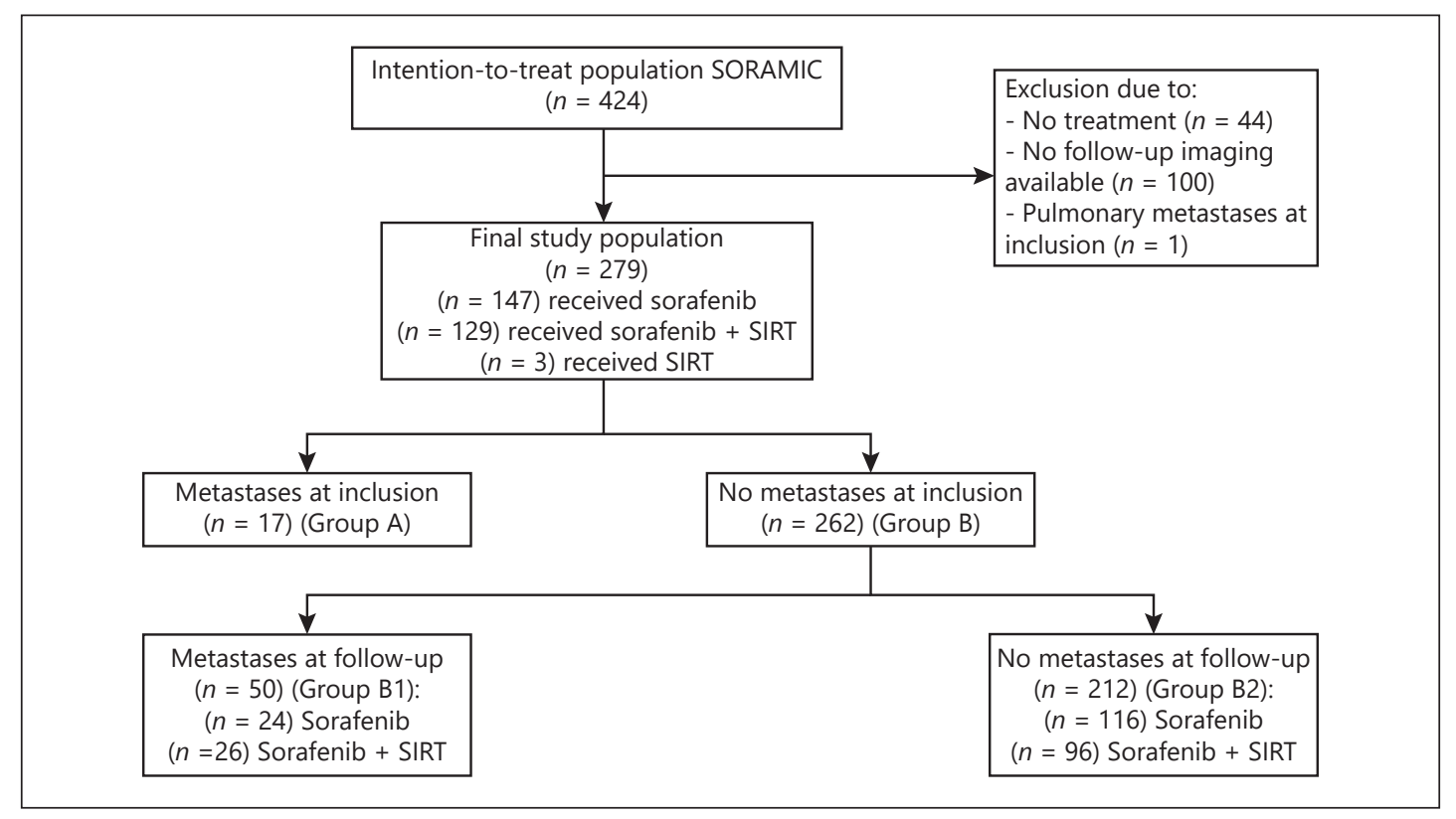

Fig. 1. Consort diagram.

of SIRT and sorafenib, and 3 patients treated with SIRT alone. This represents the safety population of the primary publication, excluding 100 patients with incomplete follow-up imaging and 1 patient with pulmonary metastases at study entry [8] (for details, refer to Fig. 1). For baseline patient characteristics, see Table 1.

In a first analysis, patients with extrahepatic organ metastases at study entry (Group A) were compared to patients without extrahepatic metastases at baseline (Group B) with respect to overall survival, regardless of follow-up staging results. Metastases to lymph nodes were not considered as extrahepatic metastases for this analysis as portal lymphadenopathy is a frequent finding in patients with chronic liver disease with a prevalence of up to $42.2 \%$ [20-22].

A second analysis, in the patients who presented without extrahepatic metastases at baseline, evaluated the impact on survival of new extrahepatic metastases in follow-up examinations (Group B1) compared to absence of extrahepatic spread (Group B2). The influence of extrahepatic spread in general and with respect to specific sites of metastases was considered.

In this analysis, any extrahepatic progression during follow-up was considered a follow-up metastasis. If the location of extrahepatic progression was not recorded in the study case report form, imaging data were re-read by an independent radiologist to determine the site, if possible. If enlarged lymph nodes evolve during palliative treatment, the probability of metastases to these nodes is much higher and can be judged as metastatic disease with reasonably high confidence. Therefore, patients who developed enlarged lymph nodes during treatment in palliative intention were judged as being progressive with new extrahepatic disease and included into the second step of the analysis.

\section{Statistical Analyses}

All statistical analyses were performed using SAS Version 9.4 for Windows (Copyright SAS Institute Inc., Cary, NC, USA). Numerical data are presented as means with standard deviations. For categorical data, results are given as absolute numbers with percentages. For comparison of categorical data, $\chi^{2}$ tests were applied. $T$ tests or Mann-Whitney $U$ tests were used for testing homogeneity of independent samples in continuous data.

For analyses of factors impacting on development of metastases during follow-up, a stepwise variable selection was used for the logistic regression model. Covariates with a high number of missing values, such as the hepatoma arterial-embolization prognostic (HAP) score, were not considered for the model, as this would decrease the already small sample size. The dataset for this analysis was therefore reduced to 44 patients with follow-up progression versus 200 patients with no metastases during follow-up. The param- 


\begin{tabular}{l|l}
\hline \multicolumn{2}{l}{ Liver Cancer 2020;9:771-786 } \\
\hline DOI: 10.1159/000510798 & $\begin{array}{l}\text { @ 2020 The Author(s). Published by S. Karger AG, Basel } \\
\text { www.karger.com/lic }\end{array}$ \\
\hline
\end{tabular}

Table 1. Baseline characteristics of cohort comparing patients with distant metastases at study entry to patients without distant metastases

\begin{tabular}{|c|c|c|c|c|}
\hline Variable & $\begin{array}{l}\text { Total } \\
n=279\end{array}$ & $\begin{array}{l}\text { Baseline metastases } \\
n=17 \text { (group A) }\end{array}$ & $\begin{array}{l}\text { No baseline metastases } \\
n=262 \text { (group B) }\end{array}$ & $p$ value \\
\hline \multicolumn{5}{|l|}{ Treatment, $n(\%)$} \\
\hline SIRT & $3(1.1)$ & $3(17.6)$ & 0 & $<0.001$ \\
\hline SIRT + sorafenib & $129(46.2)$ & $7(41.2)$ & $122(46.6)$ & \\
\hline Sorafenib & $147(52.7)$ & $7(41.2)$ & $140(53.4)$ & \\
\hline \multicolumn{5}{|l|}{ Sex, $n(\%)$} \\
\hline Female & $34(12.2)$ & $2(11.8)$ & $32(12.2)$ & 0.9563 \\
\hline Male & $245(87.8)$ & $15(88.2)$ & $230(87.8)$ & \\
\hline \multicolumn{5}{|l|}{ Age } \\
\hline Mean (SD) & $65.7(8.3)$ & $61.3(8.4)$ & $66.0(8.2)$ & 0.0230 \\
\hline Median (IQR) & $66.0(12.0)$ & $60.0(10.0)$ & $66.0(12.0)$ & \\
\hline \multicolumn{5}{|c|}{ Age category, years } \\
\hline$<65$ & $124(44.4 \%)$ & $10(58.8 \%)$ & $114(43.5 \%)$ & 0.2182 \\
\hline$\geq 65$ & $155(55.6 \%)$ & $7(41.2 \%)$ & $148(56.5 \%)$ & \\
\hline \multicolumn{5}{|l|}{$\overline{B M I}$} \\
\hline Missing & $4(1.4 \%)$ & & $4(1.5 \%)$ & 0.4317 \\
\hline Mean (SD) & $27.5(4.6)$ & $26.7(3.7)$ & $27.6(4.6)$ & \\
\hline Median (IQR) & $26.9(5.4)$ & $26.3(5.0)$ & $27.0(5.4)$ & \\
\hline \multicolumn{5}{|l|}{$E C O G, n(\%)$} \\
\hline Missing & $4(1.4)$ & & $4(1.5)$ & 0.6820 \\
\hline 0 & $202(73.5)$ & $14(82.4)$ & $188(72.9)$ & \\
\hline 1 & $72(26.2)$ & 3 (17.6) & 69 (26.7) & \\
\hline 2 & $1(0.4)$ & 0 & $1(0.4)$ & \\
\hline \multicolumn{5}{|c|}{ Liver cirrhosis, $n(\%)$} \\
\hline Missing & $3(1.1)$ & 0 & $3(1.1)$ & 0.0507 \\
\hline No & $49(17.8)$ & $6(35.3)$ & $43(16.6)$ & \\
\hline Yes & $227(82.2)$ & $11(64.7)$ & $216(83.4)$ & \\
\hline \multicolumn{5}{|l|}{ Child-Pugh, $n$ (\%) } \\
\hline A & $262(93.9)$ & $17(100)$ & $245(93.5)$ & 0.2785 \\
\hline B & $17(6.1)$ & 0 & $17(6.6)$ & \\
\hline \multicolumn{5}{|l|}{ Hepatitis B, $n(\%)$} \\
\hline No & $254(91.0)$ & $16(94.1)$ & $239(90.8)$ & 0.6466 \\
\hline Yes & $25(9.0)$ & $1(5.9)$ & $24(9.2)$ & \\
\hline \multicolumn{5}{|l|}{ Hepatitis $C, n(\%)$} \\
\hline No & $206(73.8)$ & $11(64.7)$ & $195(74.4)$ & 0.3768 \\
\hline Yes & $73(26.2)$ & $6(35.3)$ & $67(25.6)$ & \\
\hline \multicolumn{5}{|l|}{ Alcohol, n (\%) } \\
\hline No & $156(55.9)$ & $13(76.5)$ & $143(54.6)$ & 0.0781 \\
\hline Yes & $123(44.1)$ & $4(23.5)$ & $119(45.4)$ & \\
\hline \multicolumn{5}{|c|}{ Previous therapies, $n$ (\%) } \\
\hline TACE & $73(26.2)$ & $4(23.5)$ & $69(26.3)$ & 0.7986 \\
\hline $\mathrm{TAE}$ & $3(1.1)$ & 0 & $3(1.1)$ & 0.6573 \\
\hline Resection & $34(12.2)$ & $2(11.8)$ & $32(12.2)$ & 0.9563 \\
\hline RFA & $28(10.0)$ & 0 & $28(10.7)$ & 0.1553 \\
\hline Brachytherapy & $5(1.8)$ & 0 & $5(1.9)$ & 0.5655 \\
\hline
\end{tabular}


Table 1 (continued)

\begin{tabular}{|c|c|c|c|c|}
\hline Variable & $\begin{array}{l}\text { Total } \\
n=279\end{array}$ & $\begin{array}{l}\text { Baseline metastases } \\
n=17 \text { (group A) }\end{array}$ & $\begin{array}{l}\text { No baseline metastases } \\
n=262 \text { (group B) }\end{array}$ & $p$ value \\
\hline \multicolumn{5}{|c|}{ Number of lesions, $n(\%)$} \\
\hline Missing & $3(1.1)$ & 0 & $3(1.1)$ & 0.5781 \\
\hline 1 & $45(16.3)$ & $3(17.6)$ & $42(16.2)$ & \\
\hline 2 & $34(12.3)$ & $2(11.8)$ & $32(12.4)$ & \\
\hline 3 & $23(8.3)$ & $1(5.9)$ & $22(8.5)$ & \\
\hline 4 & $10(3.6)$ & $2(11.8)$ & $8(3.1)$ & \\
\hline $5-10$ & $27(9.8)$ & $2(11.8)$ & $25(9.7)$ & \\
\hline$>10$ & $137(49.6)$ & $7(41.2)$ & $130(50.2)$ & \\
\hline \multicolumn{5}{|c|}{ Max. diameter of largest lesion } \\
\hline Missing & $12(4.3 \%)$ & 0 & $12(4.6 \%)$ & 0.0605 \\
\hline Mean (SD) & $66.2(40.5)$ & $84.0(48.6)$ & $65.0(39.7)$ & \\
\hline Median (IQR) & $57.0(56.0)$ & $79.0(47.0)$ & $56.0(55.0)$ & \\
\hline \multicolumn{5}{|c|}{ Portal vein infiltration, $n(\%)$} \\
\hline Yes & $122(43.7)$ & $5(29.4)$ & $117(44.7)$ & 0.2195 \\
\hline \multicolumn{5}{|c|}{ Site of distant organ metastases, $n(\%)$} \\
\hline Bone & $11(3.9)$ & $11(64.7)$ & 0 & \\
\hline Adrenal gland & $5(1.8)$ & $5(29.4)$ & 0 & \\
\hline Peritoneum & $1(0.4)$ & $1(5.9)$ & 0 & \\
\hline \multicolumn{5}{|c|}{ BCLC stage, $n(\%)$} \\
\hline A & $5(1.8)$ & 0 & $5(1.9)$ & 0.1716 \\
\hline $\mathrm{B}$ & $85(30.5)$ & $2(11.8)$ & $83(31.7)$ & \\
\hline $\mathrm{C}$ & $189(67.7)$ & $15(88.2)$ & $174(66.4)$ & \\
\hline \multicolumn{5}{|l|}{ HAP score, $n(\%)$} \\
\hline Missing & $66(23.7)$ & $4(23.5)$ & $62(23.7)$ & 0.3043 \\
\hline 1 & $110(51.6)$ & $5(38.5)$ & $105(52.5)$ & \\
\hline 2 & $74(34.7)$ & $4(30.8)$ & $70(35.0)$ & \\
\hline 3 & $23(10.8)$ & $3(23.1)$ & $20(10)$ & \\
\hline 4 & $6(2.8)$ & $1(7.7)$ & $5(2.5)$ & \\
\hline \multicolumn{5}{|l|}{$A F P, n g / m L$} \\
\hline Missing & $14(5.0 \%)$ & 0 & $14(5.3 \%)$ & 0.8502 \\
\hline$<400$ & $177(66.8 \%)$ & $11(64.7 \%)$ & $166(66.9 \%)$ & \\
\hline$\geq 400$ & $88(33.2 \%)$ & $6(35.3 \%)$ & $82(33.1 \%)$ & \\
\hline \multicolumn{5}{|c|}{ Up-to-7 criterion, $n(\%)$} \\
\hline Missing & $13(4.7)$ & 0 & $13(5.0)$ & 0.0730 \\
\hline Inside & $40(15.0)$ & 0 & $40(16.1)$ & \\
\hline Outside & $226(85.0)$ & $17(100)$ & 209 (83.9) & \\
\hline \multicolumn{5}{|c|}{ Duration of follow-up, months } \\
\hline Missing & $4(1.4 \%)$ & 0 & $4(1.5 \%)$ & 0.7854 \\
\hline Mean (SD) & $13.9(9.6)$ & $16.1(15.1)$ & $13.8(9.1)$ & \\
\hline Median (IQR) & $11.8(12.0)$ & $9.9(15.5)$ & $12.0(11.7)$ & \\
\hline
\end{tabular}

AFP, alpha-fetoprotein; BCLC, Barcelona Clinic Liver Cancer; ECOG, Eastern Cooperative Oncology Group; HAP, hepatoma arterial-embolization prognostic; RFA, radiofrequency ablation; SIRT, selective internal radiation treatment; TACE, transarterial chemoembolization; TAE, transarterial embolization. 


\begin{tabular}{|c|c|}
\hline \multicolumn{2}{|c|}{ Liver Cancer 2020;9:771-786 } \\
\hline DOI: $10.1159 / 000510798$ & $\begin{array}{l}\text { (c) } 2020 \text { The Author(s). Published by S. Karger AG, Basel } \\
\text { www.karger.com/lic }\end{array}$ \\
\hline
\end{tabular}

Table 2. Baseline characteristics of patients who developed distant metastases during follow-up compared to those who did not

\begin{tabular}{|c|c|c|c|c|}
\hline Variable & $\begin{array}{l}\text { Total } \\
n=262\end{array}$ & $\begin{array}{l}\text { Follow-up } \\
\text { metastases } \\
n=50 \text { (group B1) }\end{array}$ & $\begin{array}{l}\text { No follow-up } \\
\text { metastases } \\
n=212 \text { (group B2) }\end{array}$ & $p$ value \\
\hline $\begin{array}{l}\text { Treatment, } n(\%) \\
\text { SIRT + sorafenib } \\
\text { Sorafenib }\end{array}$ & $\begin{array}{l}122(46.6) \\
140(53.4)\end{array}$ & $\begin{array}{l}26(52.0) \\
24(48.0)\end{array}$ & $\begin{array}{r}96(45.3) \\
116(54.7)\end{array}$ & 0.3917 \\
\hline $\begin{array}{l}\text { Sex, } n(\%) \\
\text { Female } \\
\text { Male }\end{array}$ & $\begin{array}{r}32(12.2) \\
230(87.8)\end{array}$ & $\begin{aligned} 5 & (10.0) \\
45 & (90)\end{aligned}$ & $\begin{array}{r}27(12.7) \\
185(87.3)\end{array}$ & 0.5951 \\
\hline $\begin{array}{l}\text { Age } \\
\text { Mean (SD) } \\
\text { Median (IQR) }\end{array}$ & $\begin{array}{l}66.0(8.2) \\
66.0(12.0)\end{array}$ & $\begin{array}{l}64.8(6.9) \\
65.5(10.0) \\
\end{array}$ & $\begin{array}{l}66.3(8.5) \\
66.0(13.0) \\
\end{array}$ & 0.2230 \\
\hline $\begin{array}{l}\text { Age category, years } \\
<65 \\
\geq 65\end{array}$ & $\begin{array}{l}114(43.5 \%) \\
148(56.5 \%)\end{array}$ & $\begin{array}{l}21(42.0 \%) \\
29(58.0 \%)\end{array}$ & $\begin{array}{r}93(43.9 \%) \\
119(56.1 \%) \\
\end{array}$ & 0.8106 \\
\hline $\begin{array}{l}B M I \\
\text { Missing } \\
\text { Mean (SD) } \\
\text { Median (IQR) }\end{array}$ & $\begin{array}{l}\quad 4(1.5 \%) \\
27.6(4.6) \\
27.0(5.4)\end{array}$ & $\begin{array}{c}0 \\
27.4(4.2) \\
26.8(4.7)\end{array}$ & $\begin{array}{l}\quad 4(1.9 \%) \\
27.6(4.7) \\
27.1(6.0)\end{array}$ & 0.7466 \\
\hline $\begin{array}{l}\text { ECOG, } n(\%) \\
\text { Missing } \\
0 \\
1 \\
2\end{array}$ & $\begin{array}{c}4(1.5) \\
188(72.9) \\
69(26.7) \\
1(0.4)\end{array}$ & $\begin{array}{l}0 \\
33(66.0) \\
17(34.0) \\
0\end{array}$ & $\begin{aligned} 4 & (1.9) \\
155 & (74.5) \\
52 & (25.0) \\
1 & (0.5)\end{aligned}$ & 0.3940 \\
\hline $\begin{array}{l}\text { Liver cirrhosis, } n(\%) \\
\text { Missing } \\
\text { No } \\
\text { Yes }\end{array}$ & $\begin{array}{c}3(1.1) \\
43(16.6) \\
216(83.4)\end{array}$ & $\begin{array}{c}1(2.0) \\
10(20.4) \\
39(79.6)\end{array}$ & $\begin{array}{c}2(0.9) \\
33(15.7) \\
177(84.3)\end{array}$ & 0.4265 \\
\hline $\begin{array}{l}\text { Child-Pugh, } n(\%) \\
\text { A } \\
\text { B }\end{array}$ & $\begin{array}{c}245(93.5) \\
17(6.5)\end{array}$ & $\begin{array}{c}48(96.0) \\
2(4.0)\end{array}$ & $\begin{array}{c}197(92.9) \\
15(7.1)\end{array}$ & 0.4271 \\
\hline $\begin{array}{l}\text { Hepatitis } B, n(\%) \\
\text { No } \\
\text { Yes }\end{array}$ & $\begin{array}{c}238(90.8) \\
24(9.2)\end{array}$ & $\begin{array}{c}46(92.0) \\
4(8.0)\end{array}$ & $\begin{array}{c}192(90.6) \\
20(9.4)\end{array}$ & 0.7519 \\
\hline $\begin{array}{l}\text { Hepatitis C, } n(\%) \\
\text { No } \\
\text { Yes }\end{array}$ & $\begin{array}{r}195(74.4) \\
67(25.6)\end{array}$ & $\begin{array}{l}32(64.0) \\
18(36.0)\end{array}$ & $\begin{array}{r}163(76.9) \\
49(23.1)\end{array}$ & 0.0603 \\
\hline $\begin{array}{l}\text { Alcohol, } n(\%) \\
\text { No } \\
\text { Yes }\end{array}$ & $\begin{array}{l}143(54.6) \\
119(45.4)\end{array}$ & $\begin{array}{l}33(66.0) \\
17(34.0)\end{array}$ & $\begin{array}{l}110(51.9) \\
102(48.1)\end{array}$ & 0.0714 \\
\hline $\begin{array}{l}\text { Previous therapies, } n(\%) \\
\text { TACE } \\
\text { TAE } \\
\text { Resection } \\
\text { RFA } \\
\text { Brachytherapy }\end{array}$ & $\begin{aligned} 69 & (26.3) \\
3 & (1.1) \\
32 & (12.2) \\
28 & (10.7) \\
5 & (1.9)\end{aligned}$ & $\begin{array}{l}18(36.0) \\
1(2.0) \\
5(10.0) \\
6(12.0) \\
0\end{array}$ & $\begin{aligned} 51 & (24.1) \\
2 & (0.9) \\
27 & (12.7) \\
22 & (10.4) \\
5 & (2.4)\end{aligned}$ & $\begin{array}{l}0.0846 \\
0.5276 \\
0.5951 \\
0.7383 \\
0.2729\end{array}$ \\
\hline
\end{tabular}


Table 2 (continued)

\begin{tabular}{|c|c|c|c|c|}
\hline Variable & $\begin{array}{l}\text { Total } \\
n=262\end{array}$ & $\begin{array}{l}\text { Follow-up } \\
\text { metastases } \\
n=50 \text { (group B1) }\end{array}$ & $\begin{array}{l}\text { No follow-up } \\
\text { metastases } \\
n=212(\text { group B2) }\end{array}$ & $p$ value \\
\hline \multicolumn{5}{|c|}{ Number of lesions, $n(\%)$} \\
\hline Missing & $3(1.1)$ & $1(2.0)$ & $2(0.9)$ & 0.8347 \\
\hline 1 & $42(16.2)$ & $8(16.3)$ & $34(16.2)$ & \\
\hline 2 & $32(12.4)$ & $4(8.2)$ & $28(13.3)$ & \\
\hline 3 & $22(8.5)$ & $6(12.2)$ & $16(7.6)$ & \\
\hline 4 & $8(3.1)$ & $1(2.0)$ & $7(3.3)$ & \\
\hline $5-10$ & $25(9.7)$ & $5(10.2)$ & $20(9.5)$ & \\
\hline$>10$ & $130(50.2)$ & $25(51.0)$ & $105(50)$ & \\
\hline \multicolumn{5}{|c|}{ Max. diameter of largest lesion } \\
\hline Missing & $12(4.6 \%)$ & $5(10.0 \%)$ & $7(3.3 \%)$ & 0.0369 \\
\hline Mean (SD) & $65.0(39.7)$ & $76.1(41.9)$ & $62.5(38.9)$ & \\
\hline Median (IQR) & $56.0(55.0)$ & $70.0(59.0)$ & $54.0(52.0)$ & \\
\hline \multicolumn{5}{|c|}{ Portal vein infiltration, $n(\%)$} \\
\hline Yes & $117(44.7)$ & $25(50)$ & $92(43.3)$ & 0.3982 \\
\hline \multicolumn{5}{|c|}{ Site of new extrahepatic metastases during follow-up (multiple sites possible), $n(\%)$} \\
\hline Adrenal gland & $9(3.4$ & $9(18.0)$ & 0 & \\
\hline Bone & $7(2.7)$ & $7(14.0)$ & 0 & \\
\hline Pulmonary & $13(5.0)$ & $13(26.0)$ & 0 & \\
\hline Nodal & $19(7.3)$ & $19(38)$ & 0 & \\
\hline Peritoneal & $1(0.4)$ & $1(2)$ & 0 & \\
\hline Splenic & $2(0.8)$ & $2(4)$ & 0 & \\
\hline \multicolumn{5}{|c|}{ BCLC stage, $n(\%)$} \\
\hline A & $5(1.9)$ & 0 & $5(2.4)$ & 0.0120 \\
\hline $\mathrm{B}$ & $83(31.7)$ & $8(16.0)$ & $75(35.4)$ & \\
\hline $\mathrm{C}$ & $174(66.4)$ & $42(84.0)$ & $132(62.3)$ & \\
\hline \multicolumn{5}{|c|}{ HAP score, $n(\%)$} \\
\hline Missing & $62(23.7)$ & $10(20.0)$ & $52(24.5)$ & 0.7269 \\
\hline 1 & $105(52.5)$ & $20(50)$ & $85(53.1)$ & \\
\hline 2 & $70(35)$ & $14(35)$ & $56(35)$ & \\
\hline 3 & $20(10)$ & $4(10)$ & $16(10)$ & \\
\hline 4 & $5(2.5)$ & $2(5.0)$ & 3 (1.9) & \\
\hline \multicolumn{5}{|l|}{$A F P, n g / m L$} \\
\hline Missing & $14(5.3 \%)$ & $4(8.0 \%)$ & $10(4.7 \%)$ & 0.1881 \\
\hline$<400$ & $166(66.9 \%)$ & 27 (58.7\%) & $139(68.8 \%)$ & \\
\hline$\geq 400$ & $82(33.1 \%)$ & $19(41.3 \%)$ & $63(31.2 \%)$ & \\
\hline \multicolumn{5}{|c|}{ Up-to-7 criterion, $n(\%)$} \\
\hline Missing & $13(5.0)$ & $3(6)$ & $10(4.7)$ & 0.2607 \\
\hline Inside & $40(16.1)$ & $5(10.6)$ & 35 (17.3) & \\
\hline Outside & 209 (83.9) & $42(89.4)$ & $167(82.7)$ & \\
\hline \multicolumn{5}{|c|}{ Duration of follow-up, months } \\
\hline Missing & $4(1.5 \%)$ & 0 & $4(1.9 \%)$ & 0.5604 \\
\hline Mean (SD) & $13.8(9.1)$ & $14.5(10.1)$ & $13.6(8.9)$ & \\
\hline Median (IQR) & $12.0(11.7)$ & $13.0(9.9)$ & $11.9(12.1)$ & \\
\hline
\end{tabular}

AFP, alpha-fetoprotein; BCLC, Barcelona Clinic Liver Cancer; ECOG, Eastern Cooperative Oncology Group; HAP, hepatoma-arterial embolization prognostic; RFA, radiofrequency ablation; SIRT, selective internal radiation treatment; TACE, transarterial chemoembolization; TAE, transarterial embolization. 
Schütte et al.: Impact of Metastasis on Survival in HCC

eters considered comprised treatment group, sex, age, maximum diameter of largest tumor lesion, BCLC stage, Child-Pugh stage, portal vein infiltration, Eastern Cooperative Oncology Group (ECOG) performance, presence of liver cirrhosis, and etiology of liver disease.

Finally, the impact of the site of metastases on survival was analyzed with a focus on bone and pulmonary metastases. From these analyses, all patients with an unknown site of metastases during follow-up were excluded. Overall survival was evaluated by the Kaplan-Meier method to compute nonparametric estimates of the survivor functions. Right censoring was taken into account. Survival curves were compared between both groups with the log-rank test.

All tests were carried out 2-sided. The level of significance was set to 0.05 without adjusting for multiplicity. All authors had access to the study data and reviewed and approved the final manuscript.

\section{Results}

Patient characteristics are summarized in Tables 1 and 2. Of 279 patients included in analyses, 262 (93.9\%) had no distant extrahepatic metastases at baseline, while 17 patients $(6.1 \%)$ had baseline metastases. The sites of organ metastases at study entry were bone $(n=$ $11 ; 64.7 \%)$, adrenal glands $(n=5 ; 29.4 \%)$, and peritoneum $(n=1 ; 5.9 \%)$. In patients without distant organ metastases at baseline, 31 (11.8\%) presented with enlarged lymph nodes judged to be metastases.

During follow-up of patients with no organ metastases at baseline, extrahepatic disease progression occurred in 50 patients $(19.1 \%)$, namely to adrenal glands $(n=9)$, bone $(n=7)$, lungs ( $n=13)$, lymph nodes $(n=19)$, peritoneum $(n=1)$, and spleen $(n=2)$. In 13 patients, extrahepatic disease progression was reported by the investigator without specifying the location. These images were not obtainable for re-read. In logistic regression to determine the influence of factors on development of extrahepatic disease in patients without distant organ metastases at baseline, the size of the largest tumor lesion (odd ratio [OR] 1.009 [95\% Wald CI 1.001-1.017]) and BCLC stage C versus A/B (OR 2.784 [95\% Wald CI 1.216-6.375]) had significant inputs on the risk of developing extrahepatic disease during follow-up.

The overall survival time did not differ significantly between patients with and without distant organ metastases at baseline. Patients with distant organ metastases at baseline (Group A) presented with a median survival of 11.3 months and patients without distant organ metastases at study inclusion (Group B) had a median survival of 14.8 months ( $p=$ 0.2807) (Fig. 2).

Focusing on the impact of extrahepatic progression on survival, no statistically significant difference was observed between patients without extrahepatic progression and those with new extrahepatic disease. Patients who never developed extrahepatic progression and who did not have distant organ metastases at baseline (Group B2) had a median survival of 14.8 months. In patients with extrahepatic progression (Group B1), median survival was 14.9 months ( $p=0.6483$ ) (Fig. 3a). The development of bone metastases in patients presenting without metastases at study entry did not have a statistically significant impact on median survival time (12.0 vs. 14.8 months; $p=0.6965$ ) (Fig. 3c).

However, progression with lung metastases in patients without pulmonary metastases at study entry significantly shortened both progression-free survival ( $4.7 \mathrm{vs}$. 9.4 months, $p=$ 0.0017 ) and median survival ( 7.6 vs. 15.0 months; $p=0.0060$ ) (Fig. 3b). While none of the patients who progressed with lung metastases received post-progression tumor-directed therapy, TACE was performed in 2 patients with intrahepatic progressive disease without pulmonary metastases, and 16 patients who did not develop pulmonary metastases received at least one second-line systemic therapy (11 within a clinical study, 4 received chemotherapy, and 1 received cabozantinib).

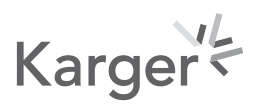


The cause of death in patients with pulmonary metastases was reported in 11 out of 13 patients. All these patients died from progressive malignant disease, while in patients with no extrahepatic metastases, the cause of death was reported in 127 cases. Out of these, 95 died from progressive disease and 32 from other causes. In 5 out of 7 patients with bone metastases the cause of death was reported. One patient died from liver failure, the others by progressive disease. Treatment modality did not have an impact on survival of patients in this analysis (Fig. 4).

\section{Discussion}

This study demonstrates that in patients with liver-dominant advanced HCC, the presence of distant organ metastases, with the exception of metastases to the lung, does not have a significant impact on treatment outcome. Even the metachronous development of extrahepatic disease while on systemic treatment does not modulate overall survival. By contrast,

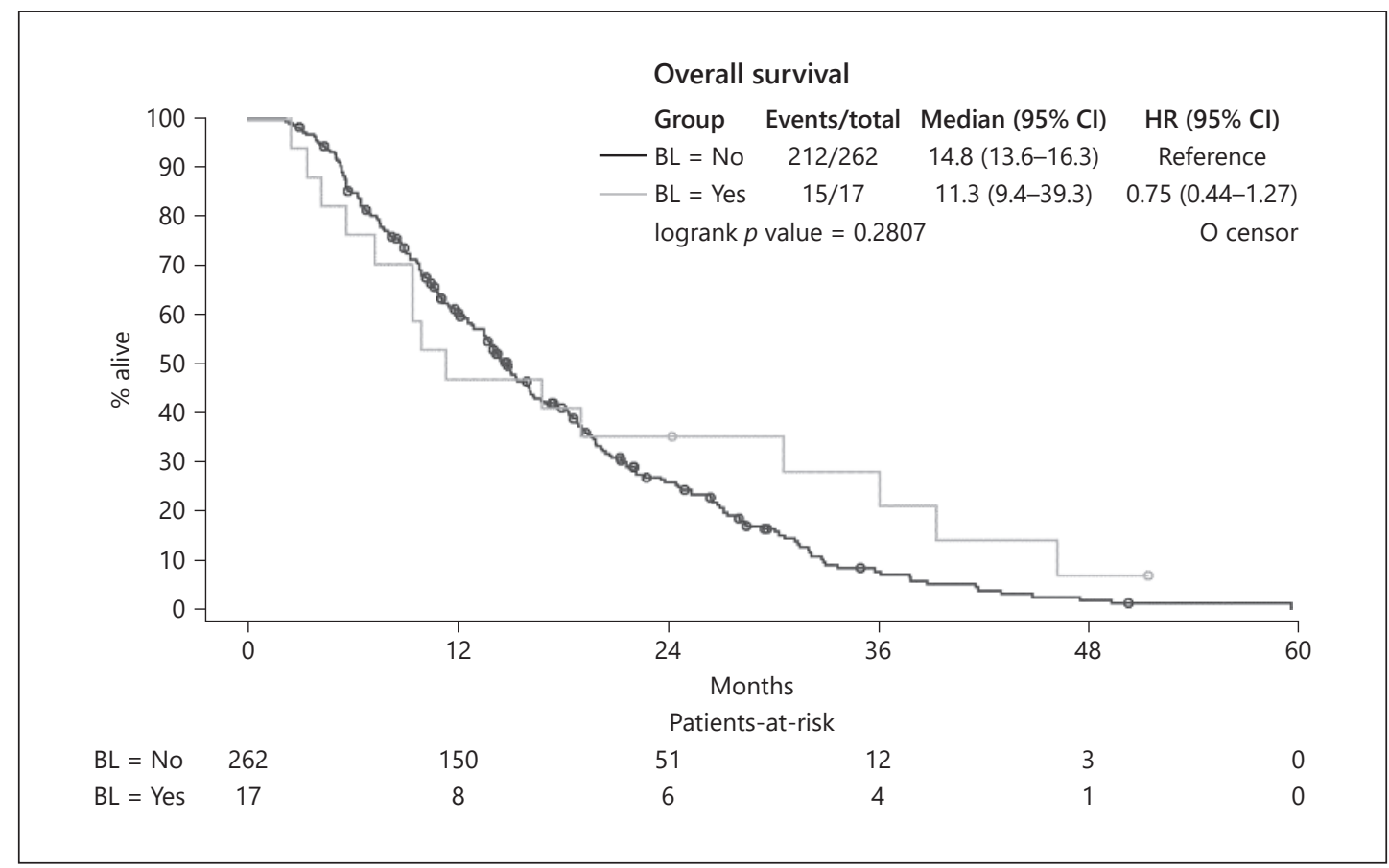

Fig. 2. Overall survival in patients with, compared to patients without, distant metastases at baseline (Groups: A vs. B). BL = No: patients without distant organ metastases at baseline; BL = Yes: patients who presented with distant organ metastases at baseline.

Fig. 3. Overall survival of patients who developed distant metastases during follow-up. a Comparison to patients who did not develop distant metastases: group B1 (FU = Yes) versus group B2 (FU = No). Patients with baseline metastases were excluded. b Influence of new pulmonary metastases on survival. Analysis includes all patients without pulmonary metastases at baseline and compares those who developed pulmonary metastases during follow-up, FU(lung) = Yes, to those who did not, FU(lung) = No. c Influence of new bone metastases on survival. Analysis includes all patients without bone metastases at study inclusion and compares those who developed bone metastases during follow-up, FU(bone) = Yes, to those who did not, FU(bone) = No. 


\begin{tabular}{|c|c|c|}
\hline \multirow[b]{2}{*}{ Liver Cancer } & \multicolumn{2}{|c|}{ Liver Cancer 2020;9:771-786 } \\
\hline & DOI: 10.1159/000510798 & $\begin{array}{l}\text { ( } 2020 \text { The Author(s). Published by S. Karger AG, Basel } \\
\text { www.karger.com/lic }\end{array}$ \\
\hline
\end{tabular}

Schütte et al.: Impact of Metastasis on Survival in HCC

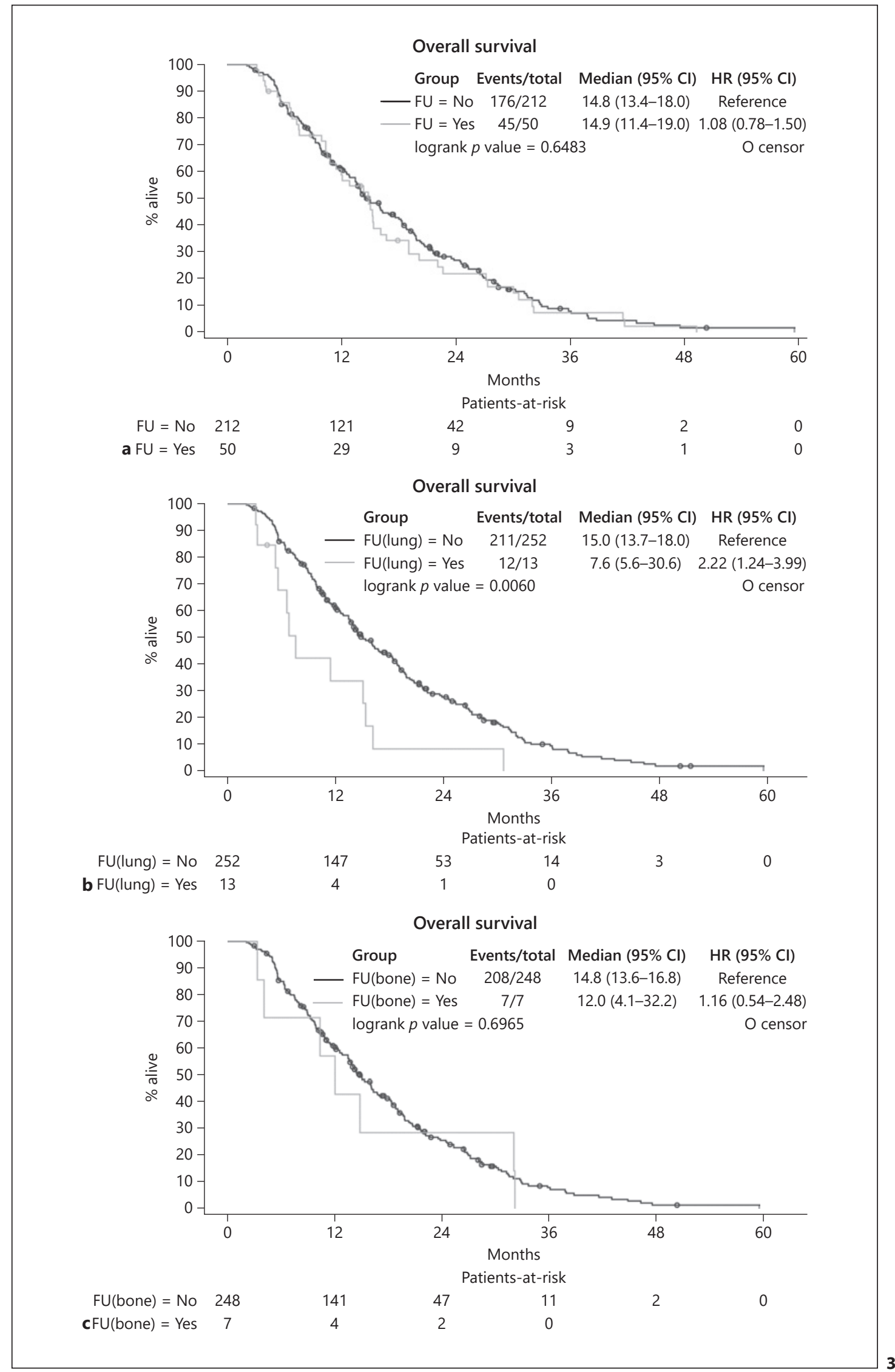




\begin{tabular}{ll|l}
\cline { 2 - 2 } Liver Cancer & \multicolumn{2}{l}{ Liver Cancer 2020;9:771-786 } \\
\cline { 2 - 3 } & DOI: 10.1159/000510798 & $\begin{array}{l}\text { @ } 2020 \text { The Author(s). Published by S. Karger AG, Basel } \\
\text { www.karger.com/lic }\end{array}$ \\
\cline { 2 - 3 }
\end{tabular}

Schütte et al.: Impact of Metastasis on Survival in HCC

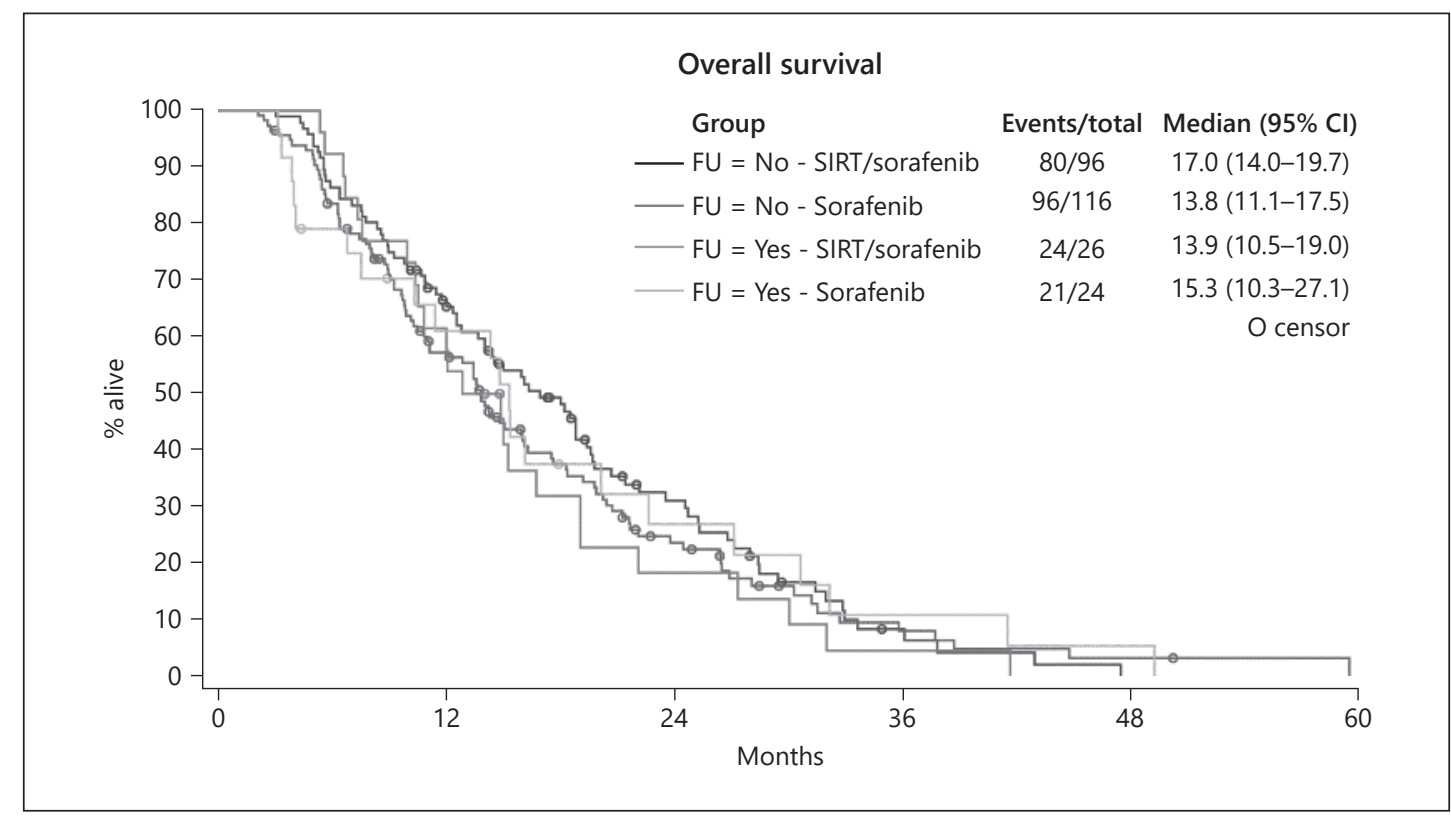

Fig. 4. Influence of treatment modality on survival in patients who developed extrahepatic metastases during follow-up.

lung metastases appear to convey a poor prognosis. The results of this study suggest that the pattern of tumor progression is a major factor impacting survival [13] in that the site of distant metastases requires special consideration. This is in line with observations from other studies reporting that in patients with HCC and extrahepatic disease, progression of the intrahepatic tumor is the cause of death in more than $80 \%$ of patients [17]. Liver failure is reported to be the cause of death in up to $89 \%$ of these patients [23]. We therefore confirm that patients with advanced HCC and progression under palliative treatment need to be treated with tailored approaches if second-line treatment modalities are evaluated.

The implementation of screening and surveillance strategies recommended by guidelines for the early detection of HCC is still insufficient even for high-risk cohorts. Accordingly, the majority of patients with HCC are diagnosed in advanced tumor stages and are not suitable for curative treatment $[1,24,25]$. Intrahepatic tumor burden, macrovascular invasion, and extrahepatic metastases, as well as severe liver function impairment, are critical factors for poor outcome $[25,26]$. The incidence rate of extrahepatic metastasis is about $2.5 \% / y e a r$ and approximately $13 \%$ at 5 years [26]. Frequent sites of extrahepatic organ spread of HCC are lungs, bones, and adrenal glands [17, 26, 27], in agreement with data we present here. In the SORAMIC trial, 17 patients had extrahepatic distant metastases at study inclusion and were considered suitable to be randomized with the option to receive additional radioembolization combined with systemic therapy. In addition, almost $20 \%$ of patients presented with new extrahepatic disease during follow-up.

Although specific subgroups (age $<65$, non-alcoholic etiology, and no cirrhosis) showed increased overall survival with SIRT plus sorafenib, in the main SORAMIC study cohort, addition of SIRT to sorafenib was not associated with improved overall survival; this was true both for the ITT and the PP subcohorts. Therefore, patients from both treatment arms were included in the present substudy, provided these treatment subgroups were balanced for parameters associated with survival differences (age, nonalcoholic etiology, and cirrhosis). 
In subgroup analyses focusing on patients with metastases, the treatment effect was also equal between both treatment arms (HR 0.70, 95\% CI 0.42-1.18; $p=0.178$ ) [8].

Extrahepatic metastases of HCC frequently lead to the decision to abstain from locoregional treatment of intrahepatic cancer $[17,28]$. Whether local tumor control in the liver in the setting of advanced disease with extrahepatic spread improves the prognosis of patients has been controversial and needs to be readdressed in the context of recently approved novel systemic drugs that allow sequential therapies in the palliative setting.

When analyzing factors that impact on prognosis in patients with advanced HCC, several aspects need to be taken into account. A subclassification of the heterogeneous BCLC stage C patients has been proposed using clinical features [29]. Based on criteria that lead to assignment to advanced tumor stage (performance status, macrovascular invasion, or extrahepatic spread), differences in median overall survival were obvious in a retrospective analysis of 835 consecutive BCLC stage C patients [30]. Others show that, in particular, the extent of portal vein invasion and the type of extrahepatic spread matter. Metastases to lymph nodes were not associated with shorter survival in a retrospective analysis from a Korean HCC registry, while the presence of organ metastases significantly impacted overall survival [31]. While the presence of macroscopic vascular invasion was confirmed to be prognostic for survival in patients with unresectable HCC in a pooled analysis of the SHARP and Asia-Pacific trials, extrahepatic spread was associated with shorter survival only in patients treated with sorafenib and in these patients predicted to have less benefit from treatment [12]. Analysis of 254 patients with HCC and extrahepatic spread treated with sorafenib confirmed, among other factors, liver function and macrovascular invasion as prognostic factors [32].

In a retrospective analysis of 240 patients with HCC and extrahepatic metastases, ChildPugh stage, smaller hepatic tumor size, absence of portal venous invasion, single metastatic organ involvement, and objective treatment response of the intrahepatic tumor were favorable prognostic factors [18]. A retrospective analysis from the SEER registry of 529 patients with HCC and extrahepatic metastases with resectable primary tumors even revealed that resection of the primary was associated with improved overall survival [33]. Patients with advanced HCC treated with sorafenib benefited from additional locoregional treatment with prolonged survival in a retrospective analysis of 312 cases [19]. Some experts have therefore stated that the majority of patients with HCC and extrahepatic metastases should undergo concurrent treatment of intrahepatic tumor manifestations, although high-quality data from prospective, randomized controlled trials to support these combined treatment approaches have been lacking [34, 35].

Several studies have focused on analysis of prognostic factors in patients progressing under systemic therapy with sorafenib. Different patterns of tumor progression have been categorized, including increase in intrahepatic/extrahepatic tumor size, development of new intrahepatic lesions, and progression with new extrahepatic lesions [13]. Progression with a new extrahepatic lesion and/or new vascular invasion is an independent predictor of postprogression survival in these studies $[14,16]$. The site of extrahepatic spread has not been taken into account in published analyses. We advise to take the site of new metastases into account in future studies, as in our analysis progression with pulmonary metastases points at a more aggressive phenotype of disease and was associated with shorter post-progression survival, while development of bone metastases was not.

In patients with HCC without extrahepatic metastases, several studies have elucidated risk factors for the development of extrahepatic disease during follow-up. These include HBsAg positivity, positive serology for hepatitis $\mathrm{C}$ virus, number and size of hepatic nodules, and vascular tumor invasion [26]. Our data confirm the risk of developing metastases in patients with large tumors and advanced disease.

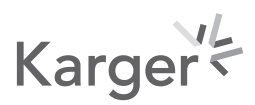


In interpreting the data presented here, several factors should be taken into account. With respect to prognosis in patients with metastases at baseline, the small number of patients may bias the analysis, as SORAMIC aimed at inclusion of patients with liver-dominant disease, while patients with pulmonary metastases were excluded by protocol. Furthermore, imaging procedures during follow-up in the palliative arm were not regulated in the study protocol, so reducing the number of patients available for this analysis. Stratification of factors significantly impacting patients' prognosis (e.g., cirrhosis vs. non-cirrhotic liver, age <65 vs. $>65$ years, alcoholic vs. nonalcoholic liver disease) would have been desirable but was not performed due to the small numbers of patients in the subgroups. Most likely, however, this would not have impacted the main findings of this study.

In conclusion, our results suggest that in patients with liver-dominant advanced HCC, metastases to distant organs, with the exception of lung metastases, do not in general exert a negative impact on prognosis and should therefore not influence the selection of first-line palliative treatments. Once progression with extrahepatic disease has occurred during palliative systemic therapy, close and personalized review of the progression pattern is mandatory in choosing second-line treatment options.

\section{Statement of Ethics}

The study was approved by the local Ethics Committees. Study procedures were performed in accordance with the protocol and ethical principles that have their origin in the Declaration of Helsinki and the International Council for Harmonisation-Good Clinical Practice. All patients provided written informed consent to participate in the study (ClinicalTrials.gov No. NCT01126645; EudraCT 2009-012576-27).

\section{Conflict of Interest Statement}

K.S.: Personal fees - Bayer. R.S.: Nothing to disclose. M.P.F.: Nothing to disclose. M.M.: Nothing to disclose. C.K.: Nothing to disclose. R.I.: Nothing to disclose. O.Ö.: Nothing to disclose. M.P.: Grants - Sirtex and Bayer; personal fees - Sirtex. B.P.: Nothing to disclose. M.S.: Personal fees - Bayer and Sirtex. R.S.: Nothing to disclose. D.P.: Grants - Sirtex and Bayer; personal fees - Sirtex and Bayer; and nonfinancial support - Sirtex. J.-P.B.: Grants - Bayer; personal fees - Bayer. P.R.: Nothing to disclose. P.M.: Nothing to disclose. J.R.: Grants - Sirtex and Bayer; personal fees - Sirtex and Bayer.

\section{Funding Sources}

SORAMIC is an investigator-initiated trial sponsored by the University of Magdeburg. Financial support was granted by Sirtex Medical and Bayer Healthcare.

\section{Author Contributions}

K.S.: Conception and design of the study; generation, collection, assembly, analysis, and/or interpretation of data; drafting or revision of the manuscript; and approval of the final version of the manuscript. R.S.: Generation, collection, assembly, analysis, and/or interpretation of data; drafting or revision of the manuscript; and approval of the final version of the manuscript. M.P.F.: Generation, collection, assembly, analysis, and/or interpretation of data; drafting or revision of the manuscript; and approval of the final version of the manuscript. M.M.: Generation, collection, assembly, analysis, and/or interpretation of data; drafting or revision of the manuscript; and approval of the final version of the manuscript. C.K.: Generation, collection, assembly, analysis, and/or interpretation of data; drafting or revision of the manuscript; and approval of the final version of the manuscript. R.I.: Generation, collection, assembly, analysis, and/or interpretation of data; drafting or revision of the manuscript; and approval of the final version of the manuscript. 0.Ö.: Generation,

\section{Karger ${ }^{\prime \prime}$}


Schütte et al.: Impact of Metastasis on Survival in HCC

collection, assembly, analysis, and/or interpretation of data; drafting or revision of the manuscript; and approval of the final version of the manuscript. M.P.: Generation, collection, assembly, analysis, and/or interpretation of data; drafting or revision of the manuscript; and approval of the final version of the manuscript. B.P.: Generation, collection, assembly, analysis, and/or interpretation of data; drafting or revision of the manuscript; and approval of the final version of the manuscript. M.S.: Generation, collection, assembly, analysis, and/or interpretation of data; drafting or revision of the manuscript; and approval of the final version of the manuscript. R.S.: Generation, collection, assembly, analysis, and/or interpretation of data; drafting or revision of the manuscript; and approval of the final version of the manuscript. D.P.: Generation, collection, assembly, analysis, and/or interpretation of data; drafting or revision of the manuscript; and approval of the final version of the manuscript. J.-P.B.: Generation, collection, assembly, analysis, and/or interpretation of data; drafting or revision of the manuscript; and approval of the final version of the manuscript. P.R.: Generation, collection, assembly, analysis, and/or interpretation of data; drafting or revision of the manuscript; and approval of the final version of the manuscript. P.M.: Conception and design of the study; generation, collection, assembly, analysis, and/or interpretation of data; drafting or revision of the manuscript; and approval of the final version of the manuscript. J.R.: Conception and design of the study; generation, collection, assembly, analysis, and/or interpretation of data; drafting or revision of the manuscript; and approval of the final version of the manuscript.

\section{References}

1 Kulik L, El-Serag HB. Epidemiology and management of hepatocellular carcinoma. Gastroenterology. 2019 Jan; 156(2):477-91.e1.

2 (IARC) GIAfRoC.

3 Schutte K, Bornschein J, Malfertheiner P. Hepatocellular carcinoma: epidemiological trends and risk factors. Dig Dis. 2009;27(2):80-92.

4 Mittal S, El-Serag HB. Epidemiology of hepatocellular carcinoma: consider the population. J Clin Gastroenterol. 2013 Jul; $47:$ S2-6.

5 European Association for the Study of the Liver. Electronic address EEE, European Association for the Study of the L. EASL clinical practice guidelines: management of hepatocellular carcinoma. J Hepatol. 2018 Jul;69(1): 182-236.

6 Galle PR, Tovoli F, Foerster F, Wörns MA, Cucchetti A, Bolondi L. The treatment of intermediate stage tumours beyond TACE: from surgery to systemic therapy. J Hepatol. 2017 Jul;67(1):173-83.

7 Llovet JM, Ricci S, Mazzaferro V, Hilgard P, Gane E, Blanc JF, et al. Sorafenib in advanced hepatocellular carcinoma. N Engl J Med. 2008 Jul 24;359(4):378-90.

8 Ricke J, Klumpen HJ, Amthauer H, Bargellini I, Bartenstein P, de Toni EN, et al. Impact of combined selective internal radiation therapy and sorafenib on survival in advanced hepatocellular carcinoma. J Hepatol. 2019 Dec;71(6):1164-74.

9 Katyal S, Oliver JH 3rd, Peterson MS, Ferris JV, Carr BS, Baron RL. Extrahepatic metastases of hepatocellular carcinoma. Radiology. 2000 Sep;216(3):698-703.

10 Natsuizaka M, Omura T, Akaike T, Kuwata Y, Yamazaki K, Sato T, et al. Clinical features of hepatocellular carcinoma with extrahepatic metastases. J Gastroenterol Hepatol. 2005 Nov;20(11):1781-7.

11 Senthilnathan S, Memon K, Lewandowski RJ, Kulik L, Mulcahy MF, Riaz A, et al. Extrahepatic metastases occur in a minority of hepatocellular carcinoma patients treated with locoregional therapies: analyzing patterns of progression in 285 patients. Hepatology. 2012 May;55(5):1432-42.

12 Bruix J, Cheng AL, Meinhardt G, Nakajima K, De Sanctis Y, Llovet J. Prognostic factors and predictors of sorafenib benefit in patients with hepatocellular carcinoma: analysis of two phase III studies. J Hepatol. 2017 Nov;67(5):999-1008.

13 Reig M, Rimola J, Torres F, Darnell A, Rodriguez-Lope C, Forner A, et al. Postprogression survival of patients with advanced hepatocellular carcinoma: rationale for second-line trial design. Hepatology. 2013 Dec;58(6):2023-31.

14 Iavarone M, Cabibbo G, Biolato M, Della Corte C, Maida M, Barbara M, et al. Predictors of survival in patients with advanced hepatocellular carcinoma who permanently discontinued sorafenib. Hepatology. 2015 Sep; 62(3):784-91.

15 Lee IC, Chen YT, Chao Y, Huo TI, Li CP, Su CW, et al. Determinants of survival after sorafenib failure in patients with BCLC-C hepatocellular carcinoma in real-world practice. Medicine. 2015 Apr;94(14):e688.

16 Ogasawara S, Chiba T, Ooka Y, Suzuki E, Kanogawa N, Saito T, et al. Post-progression survival in patients with advanced hepatocellular carcinoma resistant to sorafenib. Invest New Drugs. 2016 Apr;34(2):255-60.

17 Uchino K, Tateishi R, Shiina S, Kanda M, Masuzaki R, Kondo Y, et al. Hepatocellular carcinoma with extrahepatic metastasis: clinical features and prognostic factors. Cancer. 2011 Oct 1;117(19):4475-83.

18 Jung SM, Jang JW, You CR, Yoo SH, Kwon JH, Bae SH, et al. Role of intrahepatic tumor control in the prognosis of patients with hepatocellular carcinoma and extrahepatic metastases. J Gastroenterol Hepatol. 2012 Apr; 27(4):684-9. 
19 Sarpel U, Spivack JH, Berger Y, Heskel M, Aycart SN, Sweeney R, et al. The effect of locoregional therapies in patients with advanced hepatocellular carcinoma treated with sorafenib. HPB. 2016 May;18(5):411-8.

20 Okada Y, Yao YK, Yunoki M, Sugita T. Lymph nodes in the hepatoduodenal ligament: US appearances with CT and MR correlation. Clin Radiol. 1996 Mar;51(3):160-6.

21 Soresi M, Bonfissuto G, Magliarisi C, Riili A, Terranova A, Di Giovanni G, et al. Ultrasound detection of abdominal lymph nodes in chronic liver diseases. A retrospective analysis. Clin Radiol. 2003 May;58(5):372-7.

22 Daher S, Lev Cohen N, Massarwa M, Mahamid M, Nasser M, Hazou W, et al. Portal lymphadenopathy predicts non-alcoholic steatohepatitis and advanced fibrosis in non-alcoholic fatty liver disease. PLoS One. 2018; 13(11):e0207479.

23 Uka K, Aikata H, Takaki S, Shirakawa H, Jeong SC, Yamashina K, et al. Clinical features and prognosis of patients with extrahepatic metastases from hepatocellular carcinoma. World J Gastroenterol. 2007 Jan 21;13(3):41420.

24 Schutte K, Kipper M, Kahl S, Bornschein J, Gotze T, Adolf D, et al. Clinical characteristics and time trends in etiology of hepatocellular cancer in Germany. Digestion. 2013;87(3):147-59.

25 De Toni EN, Schlesinger-Raab A, Fuchs M, Schepp W, Ehmer U, Geisler F, et al. Age independent survival benefit for patients with hepatocellular carcinoma (HCC) without metastases at diagnosis: a population-based study. Gut. 2020. 69(1):168-76.

26 Kanda M, Tateishi R, Yoshida H, Sato T, Masuzaki R, Ohki T, et al. Extrahepatic metastasis of hepatocellular carcinoma: incidence and risk factors. Liver Int. 2008 Nov;28(9):1256-63.

27 Wu W, He X, Andayani D, Yang L, Ye J, Li Y, et al. Pattern of distant extrahepatic metastases in primary liver cancer: a SEER based study. J Cancer. 2017;8(12):2312-8.

28 Bruix J, Sherman M; Practice Guidelines Committee AAftSoLD. Management of hepatocellular carcinoma. Hepatology. 2005 Nov;42(5):1208-36.

29 Jun CH, Yoon JH, Cho E, Shin SS, Cho SB, Kim HJ, et al. Barcelona clinic liver cancer-stage C hepatocellular carcinoma: a novel approach to subclassification and treatment. Medicine. 2017 Apr;96(17):e6745.

30 Giannini EG, Bucci L, Garuti F, Brunacci M, Lenzi B, Valente M, et al. Patients with advanced hepatocellular carcinoma need a personalized management: a lesson from clinical practice. Hepatology. 2018 May;67(5): 1784-96.

31 Sinn DH, Cho JY, Gwak GY, Paik YH, Choi MS, Lee JH, et al. Different survival of Barcelona clinic liver cancer stage $C$ hepatocellular carcinoma patients by the extent of portal vein invasion and the type of extrahepatic spread. PLoS One. 2015;10(4):e0124434.

32 Sohn W, Paik YH, Cho JY, Lim HY, Ahn JM, Sinn DH, et al. Sorafenib therapy for hepatocellular carcinoma with extrahepatic spread: treatment outcome and prognostic factors. J Hepatol. 2015 May;62(5):1112-21.

33 Mao K, Yan Y, Zhang J, Wang J, Wang R, Ling X, et al. The impact of liver resection on survival outcomes of hepatocellular carcinoma patients with extrahepatic metastases: a propensity score matching study. Cancer Med. 2018 Sep;7(9):4475-84.

34 Lee HS. Management of patients with hepatocellular carcinoma and extrahepatic metastasis. Dig Dis. 2011; 29(3):333-8.

35 Finn RS, Zhu AX, Farah W, Almasri J, Zaiem F, Prokop LJ, et al. Therapies for advanced stage hepatocellular carcinoma with macrovascular invasion or metastatic disease: a systematic review and meta-analysis. Hepatology. 2018 Jan;67(1):422-35. 